\title{
Weightedness and Structural Characterization of Hierarchical Simple Games
}

\author{
Tatiana Gvozdeva, Ali Hameed and Arkadii Slinko \\ The University of Auckland, New Zealand
}

\begin{abstract}
In this paper we give structural charaterizations for disjunctive and conjunctive hierarchical simple games by characterizing them as complete games with a unique shift-maximal losing and, respectively, shift-minimal winning coalitions. We prove canonical representation theorems for both types of hierarchical games and establish duality between them. We characterize those disjunctive and conjunctive hierarchical games which are weighted majority games. This paper was inspired by Beimel et al. (2008) and Farràs and Padró (2010) characterizations of ideal weighted threshold access structures of secret sharing schemes.
\end{abstract}

Keywords:

simple game, weighted majority game, hierarchical game, duality

\section{Introduction}

In many situations cooperating agents have different status with respect to the activity they are going to undertake. To model a large class of such situations von Neumann and Morgenstern (1944) came up with a definition of a simple game which is the simplest possible type of a cooperative game, where the payoffs of coalitions are either 1 or 0 , i.e., coalitions are either "winning" or "losing". In the theory of simple games seniority of players is modeled by assigning to them different weights and setting a threshold so that a coalition is winning if the combined weight of its players is at least the threshold. This is perfectly natural, for example, in the context of corporate voting when different shareholders have different number of shares. 
The access structure in secret a sharing scheme (e.g., Simmons, 1990; Stinson, 1992) can also be modeled by a simple game, but in this theory a different approach to defining seniority is often used. To this end Simmons (1990) introduced the concept of a hierarchical access structure. Such an access structure stipulates that agents are partitioned into $m$ levels, and a sequence of thresholds $k_{1}<k_{2}<\ldots<k_{m}$ is set, so that a coalition is authorized if it has either $k_{1}$ agents of the first level or $k_{2}$ agents of the first two levels or $k_{3}$ agents of the first three levels etc. Consider, for example, the situation of a money transfer from one bank to another. If the sum to be transferred is sufficiently large, this transaction must be authorized by three senior tellers or two vice-presidents. However, two senior tellers and a vice-president can also authorize the transaction. These hierarchical structures are now called disjunctive, since only one of the $m$ conditions must be satisfied for a coalition to be authorized. If all conditions must be satisfied, then the hierarchical access structure is called conjunctive (Tassa, 2007). A typical example of a conjunctive hierarchical game would be the United Nations Security Council where for the passage of a resolution all five permanent members must vote for it and also at least nine members in total.

Both disjunctive and conjunctive hierarchical access structures have been proved to be ideal (Brickell, 1990; Tassa, 2007) which means they can carry the most informationally efficient secret sharing scheme and be completely secure (i.e., not giving any information about the secret to unauthorized coalitions). Classification of ideal access structures proved to be extremely difficult problem and the focus of attention has moved to classification of ideal access structures in subclasses of access structures like weighted threshold access structures introduced by Shamir (1979). This classification has been presented by Beimel et al. (2008) and Farràs and Padró (2010) who characterized irreducible weighted ideal access structures. All other ideal weighted threshold access structures can be obtained by combining the indecomposable ones using the operation of composition defined by Beimel et al. (2008). Their proof was indirect, the cornerstone of their approach was an application of the well-known connection between ideal secret sharing schemes and matroids (Brickell and Davenport, 1990).

In this paper we use the game theoretic methods and terminology, and we talk about hierarchical games, not access structures. We consider the classes of hierarchical disjunctive and conjunctive games which is a narrower class of games than the ideal ones. However, we give a complete description of weighted majority games in these classes, not just indecomposable ones. We 
give a direct combinatorial proof for disjunctive hierarchical games based on the technique of trading transforms developed in Taylor and Zwicker (1999).

We also characterize disjunctive hierarchical games as complete games with a unique shift-maximal losing coalitions. Then we prove the duality between disjunctive and conjunctive games. This allows us to characterize weighted conjunctive hierarchical games and obtain their structural characterization as complete games with a unique shift-minimal winning coalition. We note that the class of complete games with a unique shift-minimal winning coalition was studied in its own right in Freixas and Puente (2008) without any reference to hierarchical games.

\section{Preliminaries}

The background material on simple games can be found in Taylor and Zwicker (1999). Throughout the paper we will denote the set $\{1,2, \ldots, n\}$ as $[n]$.

Definition 1. Let $P=[n]$ be a set of players and let $\emptyset \neq W \subseteq 2^{P}$ be a collection of subsets of $P$ that satisfies the following monotonic property:

$$
\text { if } X \in W \text { and } X \subseteq Y \text {, then } Y \in W \text {. }
$$

In such case the pair $G=(P, W)$ is called a simple game and the set $W$ is called the set of winning coalitions of $G$. Coalitions that are not in $W$ are called losing.

Due to the property (1) the subset $W$ is completely determined by the set $W_{\text {min }}$ of minimal winning coalitions of $G$. A player who does not belong to any minimal winning coalition is called a dummy. Such a player can be removed from any winning coalition without making it losing.

Definition 2 (von Neumann \& Morgenstern, 1944). A simple game $G=$ $(P, W)$ is called a weighted majority game if there exist nonnegative real numbers $w_{1}, \ldots, w_{n}$, called weights, and a nonnegative real number $q$, called the threshold, such that

$$
X \in W \Longleftrightarrow \sum_{i \in X} w_{i} \geq q .
$$


In secret sharing, weighted threshold access structures were introduced by Shamir (1979).

A distinctive feature of many games is that the set of players is partitioned into subsets, and players in each of the subsets have equal status. We suggest analyzing such games with the help of multisets. Given a simple game $G$ we define a relation $\sim_{G}$ on $P$ by setting $i \sim_{G} j$ if for every set $X \subseteq P$ not containing $i$ and $j$

$$
X \cup\{i\} \in W \Longleftrightarrow X \cup\{j\} \in W .
$$

Lemma 1. (Taylor and Zwicker, 1999, Proposition 3.2.4) $\sim_{G}$ is an equivalence relation.

Example 1. Suppose we have $P=\left\{a_{1}, a_{2}, b, c\right\}$ as the set of players with weights as follows: $a_{1}$ and $a_{2}$ have weights 1 and 1.5, respectively, $b$ has weight 2 and $c$ has weight 3. Let us set the threshold $q=3$. Then the set of minimal winning coalitions for this game will be

$$
W_{\text {min }}=\left\{\left\{a_{1}, b\right\},\left\{a_{2}, b\right\},\{c\}\right\} .
$$

This gives $a_{1} \sim_{G} a_{2}, a_{i} \chi_{G} b, a_{i} \chi_{G} c, b \chi_{G} c$, and the equivalence classes are $\left\{a_{1}, a_{2}\right\},\{b\}$ and $\{c\}$.

We now need the notion of a multiset.

Definition 3. A multiset on the set $[m]$ is a mapping $\mu:[m] \rightarrow \mathbb{Z}_{+}$of $[m]$ into the set of nonnegative integers. It is often written in the form

$$
\mu=\left\{1^{k_{1}}, 2^{k_{2}}, \ldots, m^{k_{m}}\right\}
$$

where $k_{i}=\mu(i)$ is called the multiplicity of $i$ in $\mu$.

A multiset $\nu=\left\{1^{j_{1}}, \ldots, m^{j_{m}}\right\}$ is a submultiset of a multiset $\mu$ given in (4), iff $j_{i} \leq k_{i}$ for all $i \in[m]$. This is denoted as $\nu \subseteq \mu$.

The existence of large equivalence classes relative to $\sim_{G}$ allows us to compress the information about the game. This is done by the following construction. Let now $G=(P, W)$ be a game and $\sim_{G}$ be its corresponding equivalence relation. Then $P$ can be partitioned into a finite number of equivalence classes $P=P_{1} \cup P_{2} \cup \ldots \cup P_{m}$ relative to $\sim_{G}$ and suppose that $\left|P_{i}\right|=n_{i}$. Then we put in correspondence to the set of players $P$ a multiset 
$\bar{P}=\left\{1^{n_{1}}, 2^{n_{2}}, \ldots, m^{n_{m}}\right\}$. We take our base set $P$, identify those players which are equivalent and we do not distinguish between them any further. We carry over the game structure to $\bar{P}$ by defining the set of submultisets $\bar{W} \subseteq 2^{\bar{P}}$ by assuming that a submultiset $Q=\left\{1^{\ell_{1}}, 2^{\ell_{2}}, \ldots, m^{\ell_{m}}\right\}$ is winning in $\bar{G}$ if a subset of $P$ containing $\ell_{i}$ players from $P_{i}(i=1,2, \ldots, m)$, is winning in $G$. This definition is correct since the sets $P_{i}$ are defined in such a way that it does not matter which $\ell_{i}$ players from $P_{i}$ are involved. We will call $\bar{G}=(\bar{P}, \bar{W})$ the multiset representation of $G$.

Definition 4. A pair $\bar{G}=(\bar{P}, \bar{W})$ where $\bar{P}=\left\{1^{n_{1}}, 2^{n_{2}}, \ldots, m^{n_{m}}\right\}$ and $\bar{W}$ is a system of submultisets of the multiset $\bar{P}$ is said to be a simple game on $\bar{P}$ if $X \in \bar{W}$ and $X \subseteq Y$ implies $Y \in \bar{W}$.

So the multiset representation of a simple game on a set of players $P$ is a simple game on the multiset $\bar{P}$.

Given a game $G$ on a set of players $P$ we may also define a relation $\succeq_{G}$ on $P$ by setting $i \succeq_{G} j$ if for every set $X \subseteq U$ not containing $i$ and $j$

$$
X \cup\{j\} \in W \Longrightarrow X \cup\{i\} \in W .
$$

This relation is known as Isbel's desirability relation (Taylor and Zwicker, 1999). The game is called complete if $\succeq_{G}$ is a total (weak) order. We also define the relation $i \succ_{G} j$ as $i \succeq_{G} j$ but not $j \succeq_{G} i$.

Definition 5. We say that $\bar{G}=(\bar{P}, \bar{W})$ is a weighted majority game if there exist non-negative weights $w_{1}, \ldots, w_{m}$ and $q \geq 0$ such that a multiset $Q=\left\{1^{\ell_{1}}, 2^{\ell_{2}}, \ldots, m^{\ell_{m}}\right\}$ is winning iff $\sum_{i=1}^{m} \ell_{i} w_{i} \geq q$.

If $G$ is weighted, then it is well-known (see, e.g., (Taylor and Zwicker, 1999, p.91)) that we can find a weighted representation, for which equivalent players have equal weights. Hence we obtain

Proposition 1. A simple game $G=(P, W)$ is a weighted majority game if and only if the corresponding simple game $\bar{G}=(\bar{P}, \bar{W})$ is.

One of the most interesting classes of complete games is hierarchical games. They can be of two types (Beimel et al. (2008), Tassa (2007)), and they will be considered in the next section.

If a game $G$ is complete, then we define shift-minimal ( $\delta$-minimal in (Carreras and Freixas, 1996)) winning coalitions and shift-maximal losing coalitions as follows. By a shift we mean a replacement of a player of a coalition by 
a less desirable player which did not belong to it. Formally, given a coalition $X$, player $p \in X$ and another player $q \notin X$ such that $q \prec_{G} p$, we say that the coalition $(X \backslash\{p\}) \cup\{q\}$ is obtained from $X$ by a shift. A winning coalition $X$ is shift-minimal if every coalition contained in it and every coalition obtained from it by a shift are losing. A losing coalition $Y$ is said to be shift-maximal if every coalition that contains it is winning and there does not exist another losing coalition from which $Y$ can be obtained by a shift.

The definition of a shift in the multiset context must be adapted as follows.

Definition 6. Let $G$ be a complete simple game on a multiset $P=\left\{1^{n_{1}}, \ldots, m^{n_{m}}\right\}$, where $1 \succ_{G} 2 \succ_{G} \ldots \succ_{G}$ m. Suppose a submultiset

$$
A^{\prime}=\left\{\ldots, i^{\ell_{i}}, \ldots, j^{\ell_{j}}, \ldots\right\}
$$

has $\ell_{i} \geq 1$ and $\ell_{j}<n_{j}$ for some $i<j$. Then we will say that the submultiset

$$
A^{\prime}=\left\{\ldots, i^{\ell_{i}-1}, \ldots, j^{\ell_{j}+1}, \ldots\right\}
$$

is obtained from A by a shift.

Shift-minimal winning and shift-maximal losing coalitions are then defined straightforwardly.

For $X \subset P$ we will denote its complement $P \backslash X$ by $X^{c}$.

Definition 7. Let $G=(P, W)$ be a simple game and $A \subseteq P$. Let us define subsets

$$
W_{s g}=\left\{X \subseteq A^{c} \mid X \in W\right\}, \quad W_{r g}=\left\{X \subseteq A^{c} \mid X \cup A \in W\right\} .
$$

Then the game $G_{A}=\left(A^{c}, W_{s g}\right)$ is called a subgame of $G$ and $G^{A}=\left(A^{c}, W_{r g}\right)$ is called a reduced game of $G$.

It is easy to show that every subgame and every reduced game of a weighted majority game is also a weighted majority game. For example, in the case of subgame one just has to retain the same weights for elements of $A^{c}$ as in $G$ and the same threshold.

Let us briefly recap the concept of duality in games. The dual game of a game $G=(P, W)$ is defined as $G^{*}=\left(P, L^{c}\right)$. Equivalently, the winning coalitions of the game $G^{*}$ dual to $G$ are exactly the complements of losing 
coalitions of $G$. We have $G=G^{* *}$. We note also that, If $A \subseteq P$, then: $\left(G_{A}\right)^{*}=\left(G^{*}\right)^{A}$ and $\left(G^{A}\right)^{*}=\left(G^{*}\right)_{A}$. Moreover, the operation of taking the dual is known to preserve weightedness. We will also use the fact that Isbel's desirability relation is self-dual, that is $x \succeq_{G} y$ if and only if $x \succeq_{G^{*}} y$. All these concepts can be immediately reformulated for the games on multisets.

Let us remind the reader of some more facts from the theory of simple games. The sequence of an even number $2 j$ of coalitions

$$
\mathcal{T}=\left(X_{1}, \ldots, X_{j} ; Y_{1}, \ldots, Y_{j}\right)
$$

is called a trading transform if the first $j$ coalitions $X_{1}, \ldots, X_{j}$ can be converted into the second $j$ coalitions $Y_{1}, \ldots, Y_{j}$ by rearranging players. In other words, for any player $p$ the cardinality of the set $\left\{i \mid p \in X_{i}\right\}$ is the same as the cardinality of the set $\left\{i \mid p \in Y_{i}\right\}$. We say that the trading transform $\mathcal{T}$ in (6) has length $j$.

Theorem 1. (Taylor and Zwicker, 1999) A game $G=(P, W)$ is a weighted majority game if for no $j$ does there exist a trading transform (6) such that $X_{1}, \ldots, X_{j}$ are winning and $Y_{1}, \ldots, Y_{j}$ are losing.

This theorem allows to prove the existence of weights for a given game by means of a combinatorial argument.

Definition 8. Gvozdeva and Slinko, 2011) Let $G=(P, W)$ be a simple game. A trading transform (6) where all $X_{1}, \ldots, X_{j}$ are winning in $G$ and all $Y_{1}, \ldots, Y_{j}$ are losing in $G$ is called certificate of non-weightedness for $G$.

For complete games the criterion can be made easier to check, by the following result.

Theorem 2. (Freixas and Molinero, 2009) A complete game is a weighted majority game if and only if it does not have certificates of non-weightedness (6) such that $X_{1}, \ldots, X_{j}$ are shift-minimal winning coalitions and $Y_{1}, \ldots, Y_{j}$ are losing coalitions.

\section{Canonical Representations and Duality of Hierarchical Games}

Definition 9 (Disjunctive Hierarchical Game). Suppose that the set of players $P$ is partitioned into $m$ disjoint subsets $P=\cup_{i=1}^{m} P_{i}$ and let $k_{1}<k_{2}<$ 
$\ldots<k_{m}$ be a sequence of positive integers. Then we define the game $H=H_{\exists}(P, W)$ by setting

$$
W=\left\{X \in 2^{P} \mid \exists i\left(\left|X \cap\left(\cup_{j=1}^{i} P_{i}\right)\right| \geq k_{i}\right)\right\} .
$$

From the definition it follows that any disjunctive hierarchical game $H$ is complete, moreover for any $i \in[m]$ and $u, v \in P_{i}$ we have $u \sim_{H} v$. However, for arbitrary values of parameters we cannot guarantee that the canonical representation $\bar{H}$ of $H$ will be defined on the multiset $\bar{P}=\left\{1^{n_{1}}, 2^{n_{2}}, \ldots, m^{n_{m}}\right\}$, since it is possible to have less than $m$ equivalence classes. Here is an example of redundancy in the description.

Example 2. Let us consider $P=\cup_{i=1}^{3} P_{i}$ with $\left|P_{1}\right|=\left|P_{2}\right|=\left|P_{3}\right|=3$. Let us also take $\left(k_{1}, k_{2}, k_{3}\right)=(4,5,6)$ and let $H=H_{\exists}(P, W)$ be the corresponding disjunctive hierarchical game. For this game the condition $\left|X \cap P_{1}\right| \geq k_{1}$ is never satisfied. As a result we will have $x \sim_{H} y$ for every $x \in P_{1}$ and $y \in P_{2}$ leading to the multiset representation for this game on a multiset $\bar{P}=\left\{1^{6}, 2^{3}\right\}$. The same game could be obtained by taking $P=P_{1} \cup P_{2}$ with $\left|P_{1}\right|=6,\left|P_{2}\right|=3$ and $\left(k_{1}, k_{2}\right)=(5,6)$.

We see that one level of this game could be collapsed. The next theorem shows when this does not happen.

Theorem 3. Let $H=H_{\exists}(P, W)$ be a disjunctive hierarchical game defined on the set of players $P$ partitioned into $m$ disjoint subsets $P=\cup_{i=1}^{m} P_{i}$ with $n_{i}=\left|P_{i}\right|$ by a sequence of positive thresholds $k_{1}<k_{2}<\ldots<k_{m}$. Then $\sim_{H}$ has exactly $m$ equivalence classes (which are then $P_{1}, \ldots, P_{m}$ ) if and only if

(a) $k_{1} \leq n_{1}$, and

(b) $k_{i}<k_{i-1}+n_{i}$ for every $1<i<m$.

Moreover, if conditions (a) and (b) are satisfied, then $H$ contains a shiftminimal coalition of size $k_{i}$ for all $i \in[m-1]$ and $k_{1}, \ldots, k_{m-1}$ are the only possible sizes of a shift-minimal winning coalition in $H$.

Furthermore, $H$ does not have dummies if and only if $k_{m}<k_{m-1}+n_{m}$, in which case there exists a shift-minimal winning coalition consisting of $k_{m}$ players. If $k_{m} \geq k_{m-1}+n_{m}$ then $P_{m}$ consists entirely of dummies.

Proof. As was discussed earlier, the players within each $P_{i}$ are equivalent to each other. Let us prove by induction that conditions (a) and (b) imply that, for every $i \in[m-1]$, there exists a shift-minimal winning coalition $M_{i}$ of 
size $k_{i}$ that intersects with $P_{i}$ nontrivially and reaches only the $i$ th threshold. Consider the condition (a). If $k_{1} \leq n_{1}$, then any $k_{1}$ players from $P_{1}$ form a winning coalition $M_{1}$ of size $k_{1}$ which ceases to be winning if we replace one of them with a player of $P_{j}$ for every $j>1$. We now use an inductive argument. Suppose $i<m-1$. Let us now construct the shift-minimal winning coalition $M_{i+1}$. We start with subtracting an element $a \in M_{i} \cap P_{i}$ from $M_{i}$. The coalition $M_{i}^{\prime}=M_{i} \backslash\{a\}$ is losing as it does not reach any threshold. By (b) we have $k_{i+1}-k_{i}+1 \leq n_{i+1}$. This means that we can add to $M_{i}^{\prime}$ exactly $k_{i+1}-k_{i}+1$ elements of $P_{i+1}$ so that the resulting set $X$ will reach the $(i+1)$ th threshold and will therefore be winning. We now apply all possible shifts to $X$ within the set $\cup_{j=1}^{i+1} P_{j}$ and take the resulting set as $M_{i+1}$. This will secure that the coalition $M_{i+1}$ is a shift-minimal winning coalition.

It is now easy to show that $P_{i} \chi_{H} P_{j}$ for every $i \neq j$. Suppose $i<j$ and $x \in M_{i} \cap P_{i}$ and $y \in P_{j}$. Then $\left(M_{i} \backslash\{x\}\right) \cup\{y\}$ is losing and $x \succ_{H} y$ and $P_{i} \varkappa_{H} P_{j}$.

Let us now prove that if $P_{i} \chi_{H} P_{i+1}$, for all $i \in[m-1]$, then conditions (a) and (b) are satisfied. It is easy to see that $P_{1} \chi_{H} P_{2}$ implies (a). Suppose now that for $i>1$ we have $p \in P_{i}, q \in P_{i+1}$ and $p \chi_{H} q$. Then there exist $X \subseteq P$ such that $X \cup\{p\}$ is winning and $X \cup\{q\}$ is losing. This could only happen if the coalition $X \cup\{p\}$ reaches the $i$ th threshold and does not reach any other threshold, i.e., when $\left|X \cap \cup_{j=1}^{s} P_{j}\right| \leq k_{s}-1$ for $s \in[i-1]$ and $\left|X \cap \cup_{j=1}^{i} P_{j}\right|=k_{i}-1$. In particular, we have $\left|X \cap \cup_{j=1}^{i-1} P_{j}\right| \leq k_{i-1}-1$. Since $p \in P_{i} \backslash X$ we have $n_{i}-1 \geq\left|X \cap P_{i}\right| \geq k_{i}-k_{i-1}$ and (b) is proved.

It remains to prove that players of $P_{m}$ are dummies if and only if $k_{m} \geq$ $k_{m-1}+n_{m}$. If this was not true and $k_{m}<k_{m-1}+n_{m}$, arguing as above we would find a shift minimal winning coalition $M_{m}$ of cardinality $k_{m}$ that would nontrivially intersect $P_{m}$. In this case players of $P_{m}$ are not dummies. If $k_{m} \geq k_{m-1}+n_{m}$, then the last threshold is never achieved and in this case all players of $P_{m}$ are indeed dummies.

It is clear that in the case when $P_{m}$ consists of dummies we can always change the $m$ th threshold to $k_{m}=k_{m-1}+n_{m}$. We will now always do that.

Definition 10. Let $H=H_{\exists}(P, W)$ be a disjunctive hierarchical game defined on the set of players $P$ partitioned into $m$ disjoint subsets $P=\cup_{i=1}^{m} P_{i}$ with $n_{i}=\left|P_{i}\right|$ by a sequence of positive thresholds $k_{1}<k_{2}<\ldots<k_{m}$. We will say that $H$ is canonically represented if the conditions (a) and (b) of Theorem 3 are satisfied (i.e., $\sim_{H}$ has exactly $m$ equivalence classes) and $k_{m}=k_{m-1}+n_{m}$ 
in case when $P_{m}$ consists of dummies. We will denote such a game $H_{\exists}(\mathbf{n}, \mathbf{k})$, where $\mathbf{n}=\left(n_{1}, \ldots, n_{m}\right)$ and $\mathbf{k}=\left(k_{1}, \ldots, k_{m}\right)$.

If the game is canonically represented, then every level in it, except maybe the last one, adds a new class of minimal winning coalitions. We note that $\mathbf{n}=\left(n_{1}, \ldots, n_{m}\right)$ and $\mathbf{k}=\left(k_{1}, \ldots, k_{m}\right)$ in the canonical representation are uniquely defined. Indeed, the numbers $k_{1}, \ldots, k_{m-1}$ are the cardinalities of the minimal winning coalitions that exist, $k_{m}$ is a bit special but also defined uniquely.

Corollary 1. Let $G=H_{\exists}(\mathbf{n}, \mathbf{k})$ be an m-level disjunctive hierarchical game. Then we have $n_{i}>1$ for every $1<i<m$.

Proof. If $n_{i}=1$ for some $1<i<m$, then (b) cannot hold.

We note that the first and the last $m$ th level are special. If $k_{1}=1$, then every user of the first level is self-sufficient (passer) and its presence makes any coalition winning and if $k_{m} \geq k_{m-1}+n_{m}$, then the $m$ th level consists entirely of dummies.

Definition 11 (Conjunctive Hierarchical Game). Suppose that the set of players $P$ is partitioned into $m$ disjoint subsets $P=\cup_{i=1}^{m} P_{i}$, and let $k_{1}<$ $\ldots<k_{m-1} \leq k_{m}$ be a sequence of positive integers. Then we define the game $H_{\forall}(P, W)$ by setting

$$
W=\left\{X \in 2^{P} \mid \forall i\left(\left|X \cap\left(\cup_{j=1}^{i} P_{i}\right)\right| \geq k_{i}\right)\right\} .
$$

Of course, it is easy to come up with an example similar to Example 2 , so we need to look for conditions on $\mathbf{n}=\left(n_{1}, \ldots, n_{m}\right)$ and $\mathbf{k}=\left(k_{1}, \ldots, k_{m}\right)$ which guarantee that the game $H_{\forall}(P, W)$ has indeed $m$ levels.

Definition 12. Let $H=H_{\forall}(P, W)$ be a conjunctive hierarchical game defined on the set of players $P$ partitioned into $m$ disjoint subsets $P=\cup_{i=1}^{m} P_{i}$ with $n_{i}=\left|P_{i}\right|$ by a sequence of positive thresholds $k_{1}<\ldots<k_{m-1} \leq k_{m}$. We will say that $H$ is canonically represented by $\mathbf{n}=\left(n_{1}, \ldots, n_{m}\right)$ and $\mathbf{k}=\left(k_{1}, \ldots, k_{m}\right)$ if $P_{i} \nsim P_{j}$ for every distinct $i, j \in[m]$ or equivalently $\sim_{H}$ has exactly $m$ equivalence classes. We will denote such a game $H_{\forall}(\mathbf{n}, \mathbf{k})$.

As in the disjunctive case, canonical representation of a conjunctive hierarchical game is unique. 
The following result was mentioned in (Tassa, 2007, Proposition 4.1) without a proof. Since it is our main tool here we present it with a proof. In the proof it will be convenient to use multiset representations of hierarchical games.

Theorem 4. Let $\mathbf{n}=\left(n_{1}, \ldots, n_{m}\right)$ and $\mathbf{k}=\left(k_{1}, \ldots, k_{m}\right)$. Then for an $m$ level hierarchical games $H_{\exists}(\mathbf{n}, \mathbf{k})^{*}=H_{\forall}\left(\mathbf{n}, \mathbf{k}^{*}\right)$ and $H_{\forall}(\mathbf{n}, \mathbf{k})^{*}=H_{\exists}\left(\mathbf{n}, \mathbf{k}^{*}\right)$, where

$$
\mathbf{k}^{*}=\left(n_{1}-k_{1}+1, n_{1}+n_{2}-k_{2}+1, \ldots, \sum_{i \in[m]} n_{i}-k_{m}+1\right) .
$$

Proof. We will prove only the first equality. As Isbel's desirability relation is self-dual, the canonical representation of $H_{\exists}(\mathbf{n}, \mathbf{k})^{*}$ will involve the same equivalence classes and hence it will be defined on the same multiset. Let $\mathbf{k}^{*}=\left(k_{1}^{*}, k_{2}^{*}, \ldots, k_{m}^{*}\right)$. It is easy to see that $k_{i}^{*}<k_{i+1}^{*}$ is equivalent to $k_{i+1}<$ $k_{i}+n_{i+1}$ so we have $k_{1}^{*}<\ldots<k_{m-1}^{*} \leq k_{m}^{*}$ and $k_{m-1}^{*}=k_{m}^{*}$ if and only if $k_{m}=k_{m-1}+n_{m}$. So $\mathbf{k}^{*}$ is well-defined. Consider a losing coalition $X=$ $\left\{1^{\ell_{1}}, 2^{\ell_{2}}, \ldots, m^{\ell_{m}}\right\}$ in $H_{\exists}(\mathbf{n}, \mathbf{k})$. It satisfies $\sum_{j \in[i]} \ell_{j}<k_{i}$ for all $i \in[m]$. Then

$$
\sum_{j \in[i]}\left(n_{j}-\ell_{j}\right)>\sum_{j \in[i]} n_{j}-k_{i}
$$

for all $i \in[m]$, and the coalition $X^{c}=\left\{1^{n_{1}-\ell_{1}}, 2^{n_{2}-\ell_{2}}, \ldots, m^{n_{m}-\ell_{m}}\right\}$ satisfies the condition $\sum_{j \in[i]}\left(n_{j}-\ell_{j}\right) \geq \sum_{j \in[i]} n_{j}-k_{i}+1=k_{i}^{*}$, for all $i \in[m]$. Therefore, $X^{c}$ is winning in $H_{\forall}\left(\mathbf{n}, \mathbf{k}^{*}\right)$.

We need also to show that the complement of every winning in $H_{\exists}(\mathbf{n}, \mathbf{k})$ coalition is losing in $H_{\forall}\left(\mathbf{n}, \mathbf{k}^{*}\right)$. Consider a coalition $X=\left\{1^{\ell_{1}}, 2^{\ell_{2}}, \ldots, m^{\ell_{m}}\right\}$ which is winning in $H_{\exists}(\mathbf{n}, \mathbf{k})$. It means that there is an $i \in[m]$ such that $\sum_{j \in[i]} \ell_{j} \geq k_{i}$. But then the condition

$$
\sum_{j \in[i]}\left(n_{j}-\ell_{j}\right) \leq \sum_{j \in[i]} n_{j}-k_{i}<\sum_{j \in[i]} n_{j}-k_{i}+1=k_{i}^{*}
$$

holds. Thus, the complement $X^{c}=\left\{1^{n_{1}-\ell_{1}}, 2^{n_{2}-\ell_{2}}, \ldots, m^{n_{m}-\ell_{m}}\right\}$ is losing in $H_{\forall}\left(\mathbf{n}, \mathbf{k}^{*}\right)$.

We note a certain duality for the second parameter as $\mathbf{k}^{* *}=\mathbf{k}$.

For conjunctive hierarchical games the theorem analogous to Theorem 3 can be proved. 
Theorem 5. Let $H_{\forall}(P, W)$ be a conjunctive hierarchical game defined on the set of players $P$ partitioned into $m$ disjoint subsets $P=\cup_{i=1}^{m} P_{i}$, where $n_{i}=\left|P_{i}\right|$, by a sequence of positive thresholds $k_{1}<\cdots<k_{m-1} \leq k_{m}$. Then $P_{1}, \ldots, P_{m}$ are exactly the equivalence classes for $\sim_{H}$ if and only if

(a) $k_{1} \leq n_{1}$, and

(b) $k_{i}<k_{i-1}+n_{i}$ for every $1<i \leq m$.

The last mth level consists entirely of dummies if and only if $k_{m-1}=k_{m}$.

Proof. This is a direct consequence of duality and Theorem 3 .

Further we will need the following two propositions.

Proposition 2. Let $\mathbf{n}=\left(n_{1}, \ldots, n_{m}\right), \mathbf{k}=\left(k_{1}, \ldots, k_{m}\right)$ and $G=H_{\exists}(\mathbf{n}, \mathbf{k})$. If $\mathbf{n}^{\prime}=\left(n_{1}, \ldots, n_{m-1}\right), \mathbf{k}^{\prime}=\left(k_{1}, \ldots, k_{m-1}\right)$, then $H\left(\mathbf{n}^{\prime}, \mathbf{k}^{\prime}\right)$ is a subgame $G_{A}$ of $G$ for $A=\left\{m^{n_{m}}\right\}$.

Proposition 3. Let $\mathbf{n}=\left(n_{1}, \ldots, n_{m}\right), \mathbf{k}=\left(k_{1}, \ldots, k_{m}\right)$ and $G=H_{\forall}(\mathbf{n}, \mathbf{k})$. Suppose $k_{1}=n_{1}, \mathbf{n}^{\prime}=\left(n_{2}, \ldots, n_{m}\right)$, and $\mathbf{k}^{\prime}=\left(k_{2}-k_{1}, \ldots, k_{m}-k_{1}\right)$. Then $H_{\forall}\left(\mathbf{n}^{\prime}, \mathbf{k}^{\prime}\right)$ is a reduced game $G^{A}$, where $A=\left\{1^{n_{1}}\right\}$.

\section{Characterizations of Disjunctive Hierarchical Games}

First, we will obtain a structural characterization of hierarchical disjunctive games.

Theorem 6. The class of disjunctive hierarchical simple games is exactly the class of complete games with a unique shift-maximal losing coalition.

Proof. Let $G=H_{\exists}(\mathbf{n}, \mathbf{k})$ be an $m$-level hierarchical game. If $k_{m}<k_{m-1}+n_{m}$, then the following coalition is a shift-maximal losing one:

$$
M=\left\{1^{k_{1}-1}, 2^{k_{2}-k_{1}}, \ldots, m^{k_{m}-k_{m-1}}\right\} .
$$

Indeed, for every $i=1,2, \ldots, m$ it has $k_{i}-1$ players from the first $i$ levels, and so any replacement of a player with more influential one makes it winning. If $k_{m} \geq k_{m-1}+n_{m}$, then it has to be modified as

$$
M=\left\{1^{k_{1}-1}, 2^{k_{2}-k_{1}}, \ldots,(m-1)^{k_{m-1}-k_{m-2}}, m^{n_{m}}\right\} .
$$


Suppose now that $G$ is complete with the multiset representation on a multiset $P=\left\{1^{n_{1}}, 2^{n_{2}}, \ldots, m^{n_{m}}\right\}$, where $1 \succ_{G} \cdots \succ_{G} m$, and has a unique shiftmaximal losing coalition $M=\left\{1^{\ell_{1}}, 2^{\ell_{2}}, \ldots, m^{\ell_{m}}\right\}$. We claim that $\ell_{i}<n_{i}$ for all $1 \leq i<m$. Suppose not, and $\ell_{i}=n_{i}$. We know that $i \succ_{G} i+1$. It means there exists a multiset $X$ such that $X \cup\{i\}$ is winning but $X \cup\{i+1\}$ is losing. We first take $X$ to be of maximal possible cardinality, and then shift-maximal with the property $X \cup\{i\} \in W$ and $X \cup\{i+1\} \in L$. This will make $X \cup\{i+1\}$ a shift-maximal losing coalition. Indeed, we cannot add any more elements to $X$ without making it winning, also replacing any element of $X$ with a more desirable one makes it winning as well. Since $X \cup\{i+1\}$ is not equal to $M$ (the multiplicity of $i$ is not at full capacity) we get a contradiction. Hence $\ell_{i}<n_{i}$. Then $\left\{1^{\ell_{1}}, \ldots,(i-1)^{\ell_{i-1}}, i^{\ell_{i}+1}\right\}$ must be winning. Then every coalition with $k_{i}=\ell_{1}+\ldots+\ell_{i}+1$ players from the first $i$ levels is winning. Now if $\ell_{m}=n_{m}$ we set $k_{m}=k_{m-1}+n_{m}$, alternatively we set $k_{m}=\ell_{1}+\ldots+\ell_{m}+1$. It is easy to see that $G$ is in fact $H_{\exists}(\mathbf{n}, \mathbf{k})$.

Now we turn our attention to classification of weighted games in this class.

Theorem 7. Let $G=H_{\exists}(\mathbf{n}, \mathbf{k})$ be an m-level disjunctive hierarchical simple game. Then $G$ is a weighted majority game iff one of the following conditions is satisfied:

(1) $m=1$;

(2) $m=2$ and $k_{2}=k_{1}+1$;

(3) $m=2$ and $n_{2}=k_{2}-k_{1}+1$;

(4) $m \in\{2,3\}$ and $k_{1}=1$. When $m=3, G$ is weighted if and only if the subgame $H_{\exists}\left(\mathbf{n}^{\prime}, \mathbf{k}^{\prime}\right)$, where $\mathbf{n}^{\prime}=\left(n_{2}, n_{3}\right)$ and $\mathbf{k}^{\prime}=\left(k_{2}, k_{3}\right)$ falls under (2) or (3);

(5) $m \in\{2,3,4\}, k_{m} \geq k_{m-1}+n_{m}$, and the subgame $H_{\exists}\left(\mathbf{n}^{\prime}, \mathbf{k}^{\prime}\right)$, where $\mathbf{n}^{\prime}=\left(n_{1}, \ldots, n_{m-1}\right)$ and $\mathbf{k}^{\prime}=\left(k_{1}, \ldots, k_{m-1}\right)$ falls under one of the cases (1)-(4).

Proof. We will prove this theorem using the combinatorial technique of trading transforms. If $k_{m} \geq k_{m-1}+n_{m}$, then users of the last level are dummies and they never participate in any minimal winning coalition. As a result, if there exists a certificate of non-weightedness

$$
\mathcal{T}=\left(X_{1}, \ldots, X_{j} ; Y_{1}, \ldots, Y_{j}\right)
$$


with minimal winning coalitions $X_{1}, \ldots, X_{j}$, which exist by Theorem 2 , then no dummies may be found in any of the $X_{1}, \ldots, X_{j}$, hence they are not participating in this certificate. Hence $G$ is weighted if and only if its subgame $H_{\exists}\left(\mathbf{n}^{\prime}, \mathbf{k}^{\prime}\right)$, where $\mathbf{n}^{\prime}=\left(n_{1}, \ldots, n_{m-1}\right)$ and $\mathbf{k}^{\prime}=\left(k_{1}, \ldots, k_{m-1}\right)$ is weighted. So we reduce our theorem to the case without dummies, and in this case we have to prove that $G$ falls under one of the cases (1)-(4). Let us assume that $k_{m}<k_{m-1}+n_{m}$.

If $k_{1}=1$, then every user of the first level is self-sufficient (passer), that is, any coalition with participation of this player is winning. If a certificate of non-weightedness (9) exists, then a 1 cannot be a member of any set $X_{1}, \ldots, X_{j}$, since then it will have to be also in one of the $Y_{1}, \ldots, Y_{j}$ and at least one of them will not be losing. Hence $G$ is weighted if and only if its subgame $H_{\exists}\left(\mathbf{n}^{\prime}, \mathbf{k}^{\prime}\right)$, where $\mathbf{n}^{\prime}=\left(n_{2}, \ldots, n_{m}\right)$ and $\mathbf{k}^{\prime}=\left(k_{2}, \ldots, k_{m}\right)$ is weighted.

This shows that a disjunctive hierarchical simple game is weighted if and only if its subgame without passers and dummies is weighted. Hence without loss of generality we can consider games without passers and dummies. This is equivalent to assuming $k_{1}>1$ and $k_{m}<k_{m-1}+n_{m}$.

The case $m=1$ is trivial. Next we show that if at least one of the two conditions (2) and (3) is met, then $G$ is weighted. So we assume that $m=2$ and $k_{1} \geq 2$. One can easily check that each game satisfying the second condition is weighted either with weights $w_{1}=1 / k_{1}, w_{2}=1 / k_{2}\left(\right.$ if $\left.n_{2} \geq k_{2}\right)$ or $w_{1}=\frac{1}{k_{1}}, w_{2}=\frac{n_{2}-1}{k_{1} n_{2}}$ (if $n_{2}<k_{2}$ ). In the case the third condition is met, a game is weighted with weights $w_{1}=1 / k_{1}$ and $w_{2}=1 / k_{1} n_{2}$.

Conversely, we show that if all conditions (1)-(3) fail, then $G$ is not weighted. If $m=2$, this means that $k_{2} \geq k_{1}+2$ and $n_{2} \geq k_{2}-k_{1}+2$. In this case the game possesses the following certificate of non-weightedness:

$$
\left(\left\{1^{k_{1}}\right\},\left\{1^{k_{1}-2}, 2^{k_{2}-k_{1}+2}\right\} ;\left\{1^{k_{1}-1}, 2^{\left\lfloor\left(k_{2}-k_{1}+2\right) / 2\right\rfloor}\right\},\left\{1^{k_{1}-1}, 2^{\left\lceil\left(k_{2}-k_{1}+2\right) / 2\right\rceil}\right\}\right) .
$$

Since $n_{2} \geq k_{2}-k_{1}+2$, all the coalitions are well-defined. Also, the restriction $k_{2} \geq k_{1}+2$ secures that $\left\lceil\frac{k_{2}-k_{1}+2}{2}\right\rceil \leq k_{2}-k_{1}$ and makes both multisets in the right-hand-side of the trading transform losing.

Now suppose $m \geq 3, k_{1} \geq 2$. By Theorem 3 we have $k_{1} \leq n_{1}, k_{2}<k_{1}+n_{2}$ and $k_{3}<k_{2}+n_{3}$. Suppose first that $k_{3} \leq n_{3}$. Then, since $k_{3} \geq k_{2}+1 \geq$ $k_{1}+2 \geq 4$, the following is a certificate of non-weightedness.

$$
\left(\left\{1^{k_{1}}\right\},\left\{3^{k_{3}}\right\} ;\left\{1^{k_{1}-1}, 3^{2}\right\},\left\{1,3^{k_{3}-2}\right\}\right) .
$$


Suppose $k_{3}>n_{3}$. If at the same time $k_{3} \leq n_{2}+n_{3}$, then since $k_{3}-n_{3}<k_{2}$ we have a legitimate certificate of non-weightedness

$$
\left(\left\{1^{k_{1}}\right\},\left\{2^{k_{3}-n_{3}}, 3^{n_{3}}\right\} ;\left\{1^{k_{1}-1}, 2,3\right\},\left\{1,2^{k_{3}-n_{3}-1}, 3^{n_{3}-1}\right\}\right) .
$$

Finally, if $k_{3}>n_{3}$ and $k_{3}>n_{2}+n_{3}$, then the certificate of non-weightedness will be

$$
\begin{aligned}
& \left(\left\{1^{k_{1}}\right\},\left\{1^{k_{3}-n_{2}-n_{3}}, 2^{n_{2}}, 3^{n_{3}}\right\} ;\right. \\
& \left.\left\{1^{k_{1}-1}, 2,3\right\},\left\{1^{k_{3}-n_{2}-n_{3}+1}, 2^{n_{2}-1}, 3^{n_{3}-1}\right\}\right) .
\end{aligned}
$$

All we have to check is that the second coalition of the losing part is indeed losing. To show this we note that $k_{3}-n_{3}<k_{2}$ and $k_{3}-n_{2}-n_{3}+1<$ $k_{2}-n_{2}+1 \leq k_{1}$. This shows that the second coalition of the losing part is indeed losing and proves the theorem.

\section{Characterizations of Conjunctive Hierarchical Games}

First we obtain a structural characterization of conjunctive hierarchical games.

Theorem 8. The class of conjuctive hierarchical simple games is exactly the class of complete games with a unique shift-minimal winning coalition.

Proof. Let $H_{\forall}(\mathbf{n}, \mathbf{k})$ be a conjunctive hierarchical game. By Theorem 4 , the dual game of $H_{\forall}(\mathbf{n}, \mathbf{k})$ is a disjunctive hierarchical game $H_{\exists}\left(\mathbf{n}, \mathbf{k}^{*}\right)$. If we can prove that the class of complete games with a unique shift-minimal winning coalition is dual to the class of complete games with a unique shift-maximal losing coalition, then by Theorem 6 this will be sufficient.

Let $G=(P, W)$ be a simple game with the unique shift-maximal losing coalition $S$. By definition, $S^{c}$ is winning in $G^{*}$. Let us prove that it is a shift-minimal winning coalition. Consider any other coalition $X$ that can be obtained from $S^{c}$ by a shift in $G^{*}$. It means that there are players $i \in X$ and $j \notin X$ such that $j \prec_{G^{*}} i$ and $X=\left(S^{c} \backslash\{i\}\right) \cup\{j\}$. The complement of $X$ is the set $X^{c}=(S \backslash\{j\}) \cup\{i\}$. Furthermore, $j \prec_{G} i$. The coalition $X^{c}$ is winning in $G$, because there does not exist a losing coalition from which $S$ can be obtained by a shift. Therefore, $X$ is losing in $G^{*}$ and every coalition obtained from $S^{c}$ by a shift is also losing in $G^{*}$. Consider now a subset $X$ of $S^{c}$. The complement $X^{c}$ of $X$ is a superset of $S$. Hence, $X^{c}$ is winning in $G$ and $X$ is losing in $G^{*}$. Thus, $S^{c}$ is the shift-minimal winning coalition in $G^{*}$. 
We claim that $S^{c}$ is the unique shift-minimal winning coalition in $G^{*}$. Assume, to the contrary, there is another shift-minimal winning coalition $X$ in $G^{*}$. As we have seen above $X^{c}$ would be shift-maximal losing coalition in $G$ and it is different from $S$, a contradiction.

It is interesting that the class of complete games with a unique shiftminimal winning coalition was studied before Freixas and Puente (2008), without noticing that this class is actually the class of conjunctive hierarchical games.

Theorem 9. Let $G=H_{\forall}(\mathbf{n}, \mathbf{k})$ be an m-level conjunctive hierarchical simple game. Then $G$ is a weighted majority game iff one of the following conditions is satisfied:

(1) $m=1$;

(2) $m=2$ and $k_{2}=k_{1}+1$;

(3) $m=2$ and $n_{2}=k_{2}-k_{1}+1$;

(4) $m \in\{2,3\}$ and $k_{1}=n_{1}$. When $m=3, G$ is weighted if and only if the reduced game $H_{\forall}(\mathbf{n}, \mathbf{k})^{\left\{1^{n_{1}}\right\}}=H_{\forall}\left(\mathbf{n}^{\prime}, \mathbf{k}^{\prime}\right)$, where $\mathbf{n}^{\prime}=\left(n_{2}, n_{3}\right)$ and $\mathbf{k}^{\prime}=\left(k_{2}-k_{1}, k_{3}-k_{1}\right)$ falls under (2) or (3);

(5) $m \in\{2,3,4\}, k_{m}=k_{m-1}$, and the reduced game $H_{\forall}^{\left\{m^{n_{m}}\right\}}(\mathbf{n}, \mathbf{k})=$ $H_{\forall}\left(\mathbf{n}^{\prime}, \mathbf{k}^{\prime}\right)$, where $\mathbf{n}^{\prime}=\left(n_{1}, \ldots, n_{m-1}\right)$ and $\mathbf{k}^{\prime}=\left(k_{1}, \ldots, k_{m-1}\right)$ falls under one of the (1) - (4);

Proof. The theorem straightforwardly follows from Theorem 7 , the duality between conjunctive hierarchical games and disjunctive hierarchical game and Propositions 2 and 3 .

\section{Conclusion and Further Research}

It is interesting to compare our results with the results by Farràs and Padró (2010) (their classification is more accurate than the one in Beimel et al. (2008)). They list seven types of indecomposable simple games: $k$ out-of- $n$ symmetric simple games; three bipartite types $B_{1}, B_{2}, B_{3}$ and three tripartite ones $T_{1}, T_{2}, T_{3}$. The $k$-out-of- $n$ symmetric game is the case (1) of both Theorems 9 and 7. As to the rest, $B_{1}$ is conjunctive hierarchical and corresponds to case (3) of Theorems 9. Both types $B_{2}$ and $B_{3}$ correspond to subcases of case (2) of Theorem 7 . The tripartite games are neither disjunctive nor conjunctive hierarchical. 
A very natural question to ask is to characterize all weighted tripartite games as defined in Beimel et al. (2008)). Farràs and Padró (2010) characterized all weighted indecomposable tripartite simple games. Also the operation

of composition of games employed in Beimel et al. (2008)) and Farràs and Padró (2010) is not well-understood from the algorithmic point of view and there are no efficient algorithms to find the decomposition if the game is decomposable. The uniqueness of the decomposition into indecomposable games has not been proved.

One obvious conclusion that can be drawn from our research is that the two methods of modeling seniority, namely, by assigning weights and assigning a level in a hierarchy, are very different. Another conclusion is that hierarchical games of both types provide very natural examples of complete games without weighted voting representation.

It would be interesting to know how many more hierarchical games are contained in the class of roughly weighted games which was introduced in Taylor and Zwicker (1999) and studied in Gvozdeva and Slinko (2011). Roughly weighted games allow just one special value (the threshold) such that coalitions whose weight are equal to this value could be either winning or losing. A further development of this idea realized in Gvozdeva et al. (2010) stipulate an interval of special values - such games are called superroughly weighted. We have a hierarchy of super-roughly weighted games $\mathcal{C}_{\alpha}$ parameterized by a real number $\alpha$ which is the length of the interval of special values. It would be interesting to know how many levels a hierarchical game from the class $\mathcal{C}_{\alpha}$ might have.

\section{The Bibliography}

Beimel, A., Tassa, T., Weinreb, E., 2008. Characterizing ideal weighted threshold secret sharing. SIAM Journal on Discrete Mathematics 22 (1), 360-397.

Brickell, E., Davenport, D., 1990. On the classification of ideal secret sharing schemes. In: Brassard, G. (Ed.), Advances in Cryptology CRYPTO 89 Proceedings. Vol. 435 of Lecture Notes in Computer Science. Springer Berlin / Heidelberg, pp. 278-285.

Brickell, E. F., 1990. Some ideal secret sharing schemes. In: Proceedings of the workshop on the theory and application of cryptographic techniques on 
Advances in cryptology. Springer-Verlag New York, Inc., New York, NY, USA, pp. 468-475.

Carreras, F., Freixas, J., 1996. Complete simple games. Mathematical Social Sciences 32 (2), 139-155.

Farràs, O., Padró, C., 2010. Ideal hierarchical secret sharing schemes. In: Micciancio, D. (Ed.), Theory of Cryptography. Vol. 5978 of Lecture Notes in Computer Science. Springer Berlin / Heidelberg, pp. 219-236.

Freixas, J., Molinero, X., 2009. Simple games and weighted games: A theoretical and computational viepoint. Discrete Applied Mathematics 157, $1496-1508$.

Freixas, J., Puente, M. A., 2008. Dimension of complete simple games with minimum. European Journal of Operational Research 188 (2), 555-568.

Gvozdeva, T., Hemaspaandra, L., Slinko, A., 2010. Three hierarchies of simple games parameterized by "resource" parameters. In: Proceedings of the 3rd International Workshop on Computational Social Choice. pp. 259-270.

Gvozdeva, T., Slinko, A., 2011. Weighted and roughly weighted simple games. Mathematical Social Sciences 61, 20-30.

Shamir, A., 1979. How to share a secret. Commun. ACM 22, 612-613.

Simmons, G. J., 1990. How to (really) share a secret. In: Proceedings of the 8th Annual International Cryptology Conference on Advances in Cryptology. Springer-Verlag, London, UK, pp. 390-448.

Stinson, D. R., 1992. An explication of secret sharing schemes. Des. Codes Cryptography 2, 357-390.

Tassa, T., 2007. Hierarchical threshold secret sharing. J. Cryptol. 20, 237264.

Taylor, A., Zwicker, W., 1999. Simple games. Princeton University Press.

von Neumann, J., Morgenstern, O., 1944. Theory of games and economic behavior. Princeton University Press. 